\title{
Towards Development of New Materials and New Electrochemical Devices
}

\author{
Ryoji KANNO
}

In the technological development of batteries and fuel cells, the relationship between material development and device development is always problematic.

There is strong demand for new devices for batteries and fuel cells. The technological roadmap prepared by the Japanese government also points to the path of development, and systematic development is in progress worldwide.

Batteries, fuel cells, and capacitors are devices based on an electrochemical reaction, and this electrochemical reaction determines the properties of the device. For example, the capacity, voltage, and output of batteries are determined by electrochemical properties such as electrochemical potential and conductivity. Limitation characteristics of the new device can be predicted from the electrochemical reaction; thus, deep understanding of electrochemical reactions is necessary for development. At present, a good example is the new electrochemical devices (metal-air, etc.), for which we have high expectations.

On the other hand, since electrochemical devices are composed of a combination of materials with different functions such as electrodes and electrolytes, deep understanding of materials becomes necessary in order to find the optimum combination. In other words, whether the optimum properties expected for the device are actualized or not is determined by the limits of the materials. Not only the type of material, but also the state, composition, size (ranging from bulk to nano), and even shape have a significant impact. Furthermore, unpredicted materials are sometimes discovered.

As revealed by the fact that lithium batteries with expected high battery potentials and high energy densities were only actualized after the development of intercalation materials such as oxide positive electrodes and carbon negative electrodes, and electrolytes that can withstand high potentials, new materials promote innovative devices. It also indicates that new devices can only be created from new materials.

There are two routes for the development of new devices: 1) material driven-the appearance of new materials with properties different from existing materials promotes the development of new devices, and 2) electrochemical reaction driven - the development of new materials to actualize device properties predicted from electrochemical reactions.

There is an unbreakable tie between material development and device development, but it is unclear as to which takes initiative for development.

There is no answer to this question, and there is no clear and quick pathway from material development to electrochemical device construction. In fact, there should be many different pathways. From diverse research directionalities, pathways to various devices split, leading to diverse research topics and manners of progress in technological development.

This is a special issue for the 55th "Battery Symposium in Japan", held in the fall of 2014. As the importance of electrochemical devices and their materials has increased in recent years, this special issue is quite timely. At the "Battery Symposium in Japan" approximately 35 years ago, research presentations were limited to devices such as lead acid batteries, molten carbonate fuel cells, and manganese dry cells. The types of materials used were also limited. Reviewing the research topics of this special issue, I was surprised by the diversity of devices along with the development of these materials, such as how and where these diversities have spread.

In the future, with the appearance of materials from beyond the existing knowledge in addition to devices that can be developed by combining existing knowledge, new devices may become available. This introduces challenges for new science and technology beyond the range of existing knowledge on electrochemistry and materials. This is an extremely interesting time.

In this special issue, I hope you discover many research topics with potential such as those discussed above.

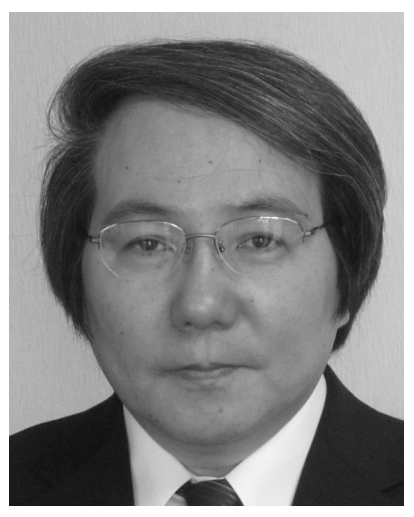

Ryoji KANNO

Former Editor in Chief,

Electrochemistry

The Committee of Battery

Technology, The Electro-

chemical Society of Japan,

Vice Chairman 